REPRESENTATION THEORY

An Electronic Journal of the American Mathematical Society

Volume 3, Pages 444-456 (November 9, 1999)

S $1088-4165(99) 00066-7$

\title{
THE ADJOINT REPRESENTATION OF A REDUCTIVE GROUP AND HYPERPLANE ARRANGEMENTS
}

\author{
J. MATTHEW DOUGLASS
}

\begin{abstract}
Let $G$ be a connected reductive algebraic group with Lie algebra $\mathfrak{g}$ defined over an algebraically closed field, $k$, with char $k=0$. Fix a parabolic subgroup of $G$ with Levi decomposition $P=L U$ where $U$ is the unipotent radical of $P$. Let $\mathfrak{u}=\operatorname{Lie}(U)$ and let $\mathfrak{z}$ denote the center of $\operatorname{Lie}(L)$. Let $T$ be a maximal torus in $L$ with Lie algebra $\mathfrak{t}$. Then the root system of $(\mathfrak{g}, \mathfrak{t})$ is a subset of $\mathfrak{t}^{*}$ and by restriction to $\mathfrak{z}$, the roots of $\mathfrak{t}$ in $\mathfrak{u}$ determine an arrangement of hyperplanes in $\mathfrak{z}$ we denote by $\mathcal{A}^{\mathfrak{z}}$. In this paper we construct an isomorphism of graded $k[\mathfrak{z}]$-modules $\operatorname{Hom}_{G}\left(\mathfrak{g}^{*}, k\left[G \times^{P}(\mathfrak{z}+\mathfrak{u})\right]\right) \cong D\left(\mathcal{A}^{\mathfrak{z}}\right)$, where $D\left(\mathcal{A}^{\mathfrak{z}}\right)$ is the $k[\mathfrak{z}]$-module of derivations of $\mathcal{A}^{\mathfrak{z}}$. We also show that $\operatorname{Hom}_{G}\left(\mathfrak{g}^{*}, k\left[G \times{ }^{P}(\mathfrak{z}+\mathfrak{u})\right]\right)$ and $k[\mathfrak{z}] \otimes \operatorname{Hom}_{G}\left(\mathfrak{g}^{*}, k\left[G \times \times^{P} \mathfrak{u}\right]\right)$ are isomorphic graded $k[\mathfrak{z}]$-modules, so $D\left(\mathcal{A}^{\mathfrak{z}}\right)$ and $k[\mathfrak{z}] \otimes \operatorname{Hom}_{G}\left(\mathfrak{g}^{*}, k\left[G \times{ }^{P} \mathfrak{u}\right]\right)$ are isomorphic, graded $k[\mathfrak{z}]$-modules. It follows immediately that $\mathcal{A}^{\mathfrak{z}}$ is a free hyperplane arrangement. This result has been proved using case-by-case arguments by Orlik and Terao. By keeping track of the gradings involved, and recalling that $\mathfrak{g}$ affords a self-dual representation of $G$, we recover a result of Sommers, Trapa, and Broer which states that the degrees in which the adjoint representation of $G$ occurs as a constituent of the graded, rational $G$-module $k\left[G \times{ }^{P} \mathfrak{u}\right]$ are the exponents of $\mathcal{A}^{\mathfrak{z}}$. This result has also been proved, again using case-by-case arguments, by Sommers and Trapa and independently by Broer.
\end{abstract}

\section{INTRODUCTION}

Suppose $k$ is an algebraically closed field with characteristic zero and $G$ is a connected, reductive, algebraic group defined over $k$ with Lie algebra $\mathfrak{g}$. Let $\mathcal{N}$ denote the cone of nilpotent elements in $\mathfrak{g}$. Then $G$ acts on $\mathfrak{g}$ by the adjoint representation and $\mathcal{N}$ is a closed, $G$-invariant subvariety, so $G$ acts on $k[\mathcal{N}]$, the ring of regular functions on $\mathcal{N}$. Since $\mathcal{N}$ is a cone, $k[\mathcal{N}]$ inherits a grading from $k[\mathfrak{g}]$ and each homogeneous component, $k[\mathcal{N}]_{j}$, is a $G$-stable subspace. Kostant [5] proved that $\mathfrak{g}$ occurs as a constituent of $k[\mathcal{N}]_{j}$ if and only if $j$ is an exponent of the Weyl group of $G$. Precisely, he proved the equality of polynomials in $q$ :

$$
\sum_{j \geq 0} \operatorname{dim} \operatorname{Hom}_{G}\left(\mathfrak{g}, k[\mathcal{N}]_{j}\right) q^{j}=q^{n_{1}}+\cdots+q^{n_{l}}
$$

where $\left\{n_{1}, \ldots, n_{l}\right\}$ is the multiset of exponents of $W$ (see $[7, \S 6.2]$ for a definition of the exponents of $W$ ).

Sommers and Trapa [9] and also Broer [4] have generalized this result as follows. Fix a maximal torus, $T$, and a parabolic subgroup, $P$, of $G$ with $T \subseteq P$. Let $U$ denote the unipotent radical of $P$ and let $L$ be a Levi subgroup of $P$ containing

Received by the editors March 8, 1999 and, in revised form September 28, 1999.

1991 Mathematics Subject Classification. Primary 22E46.

(C)1999 American Mathematical Society 
$T$. We follow the convention that lower case Fraktur letters will denote the Lie algebra of the group denoted by the same upper case Roman letter, so for example $\mathfrak{t}=\operatorname{Lie}(T)$, and $\mathfrak{u}=\operatorname{Lie}(U)$. Let $\mathfrak{z}$ denote the center of $\mathfrak{l}$, so $\mathfrak{z} \subseteq \mathfrak{t}$. Then $\mathfrak{z}$ acts on $\mathfrak{u}$ by restricting the adjoint representation of $\mathfrak{g}$ and the weights of $\mathfrak{z}$ on $\mathfrak{u}$ are the restrictions to $\mathfrak{z}$ of the weights of $\mathfrak{t}$ on $\mathfrak{u}$. The kernels of these weights determine a hyperplane arrangement in $\mathfrak{z}$ we denote by $\left(\mathfrak{z}, \mathcal{A}^{\mathfrak{z}}\right)$. Orlik and Terao $[8]$ have proven, using case-by-case arguments, that $\mathcal{A}^{\mathfrak{z}}$ is a free hyperplane arrangement and Orlik and Solomon [6] have computed the exponents of $\mathcal{A}^{\mathfrak{z}}$ in all cases.

Now $P$ acts on $\mathfrak{u}$ and so we can form the associated fibre bundle, $G \times{ }^{P} \mathfrak{u}$, over $G / P$ with fibre $\mathfrak{u}$. Then $G$ acts on $G \times^{P} \mathfrak{u}$ by left multiplication and so $G$ acts on $k\left[G \times{ }^{P} \mathfrak{u}\right]$, the ring of global regular functions on $G \times{ }^{P} \mathfrak{u}$. Moreover, $k\left[G \times{ }^{P} \mathfrak{u}\right]$ inherits a grading from the scalar action of $k$ on $\mathfrak{u}, k\left[G \times{ }^{P} \mathfrak{u}\right] \cong \bigoplus_{j \geq 0} k\left[G \times{ }^{P} \mathfrak{u}\right]_{j}$, and each homogeneous component is a $G$-stable subspace. Sommers and Trapa [9], and independently Broer [4], have proven that $\mathfrak{g}$ occurs as a constituent of $k\left[G \times{ }^{P} \mathfrak{u}\right]_{j}$ if and only if $j$ is an exponent of $\mathcal{A}^{\mathfrak{z}}$. Precisely, they prove the equality of polynomials in $q$ :

$$
\sum_{j \geq 0} \operatorname{dim} \operatorname{Hom}_{G}\left(\mathfrak{g}, k\left[G \times{ }^{P} \mathfrak{u}\right]_{j}\right) q^{j}=q^{n_{1}^{3}}+\cdots+q^{n_{r}^{3}}
$$

where $\left\{n_{1}^{\mathfrak{z}}, \ldots, n_{r}^{\mathfrak{z}}\right\}$ is the multiset of exponents of $\mathcal{A}^{\mathfrak{z}}$ as defined in [7, Definition 4.25].

In the special case when $P$ is a Borel subgroup of $G$, then $\mathfrak{z}=\mathfrak{t}$, the exponents of $\mathcal{A}^{\mathfrak{t}}$ are the exponents of the Weyl group of $G$, and $k[\mathcal{N}]_{j}$ and $k\left[G \times{ }^{P} \mathfrak{u}\right]_{j}$ afford equivalent representations of $G$ for $j \geq 0$, so we recover Kostant's result.

The proofs given by Sommers and Trapa and by Broer of the equality (1.1) are both case-by-case arguments, with separate arguments for each type of root system.

In this paper, our main result is that there is an isomorphism of graded $k[\mathfrak{z}]$ modules $\Gamma: \operatorname{Hom}_{G}\left(\mathfrak{g}^{*}, k\left[G \times{ }^{P}(\mathfrak{z}+\mathfrak{u})\right]\right) \rightarrow D\left(\mathcal{A}^{\mathfrak{z}}\right)$, where $D\left(\mathcal{A}^{\mathfrak{z}}\right)$ is the $k[\mathfrak{z}]$-module of derivations of $\mathcal{A}^{\mathfrak{z}}$. We also show that there is an isomorphism of graded $k[\mathfrak{z}]$-modules between $\operatorname{Hom}_{G}\left(\mathfrak{g}^{*}, k\left[G \times{ }^{P}(\mathfrak{z}+\mathfrak{u})\right]\right)$ and $k[\mathfrak{z}] \otimes \operatorname{Hom}_{G}\left(\mathfrak{g}^{*}, k\left[G \times{ }^{P} \mathfrak{u}\right]\right)$. Thus $D\left(\mathcal{A}^{\mathfrak{z}}\right)$ and $k[\mathfrak{z}] \otimes \operatorname{Hom}_{G}\left(\mathfrak{g}^{*}, k\left[G \times{ }^{P} \mathfrak{u}\right]\right)$ are isomorphic, graded $k[\mathfrak{z}]$-modules, so by keeping track of the gradings involved, and recalling that $\mathfrak{g}$ affords a self-dual representation of $G$, we see that the isomorphism $\Gamma$ explains the computation, (1.1), of Sommers, Trapa, and Broer.

Our proof that $\operatorname{Hom}_{G}\left(\mathfrak{g}^{*}, k\left[G \times{ }^{P}(\mathfrak{z}+\mathfrak{u})\right]\right)$ and $D\left(\mathcal{A}^{\mathfrak{z}}\right)$ are isomorphic, graded $k[\mathfrak{z}]$ modules does not involve any case-by-case computations. We define $\Gamma$ explicitly and then show, using that the set of semisimple elements is dense in $\mathfrak{g}$ and some results on the $P$-orbit structure of $\mathfrak{z}+\mathfrak{u}$, that it's a bijection.

Since $k[\mathfrak{z}] \otimes \operatorname{Hom}_{G}\left(\mathfrak{g}^{*}, k\left[G \times{ }^{P} \mathfrak{u}\right]\right)$ and $D\left(\mathcal{A}^{\mathfrak{z}}\right)$ are isomorphic $k[\mathfrak{z}]$-modules, it follows immediately that the arrangement $\mathcal{A}^{\mathfrak{z}}$ is free. We thus obtain a proof, free of case-by-case considerations, of the result of Orlik and Terao [8] that the restrictions of the reflection arrangements arising from Weyl groups are free.

After learning of our ideas about the definition of $\Gamma$, Broer [3] has given another proof that it's surjective, the difficult part of showing that it's an isomorphism. His proof is elegant, using Saito's criterion, but relies on a previous result of his concerning sums of exponents. Our proof that $\Gamma$ is surjective is completely different than Broer's and uses only some basic algebraic geometry and properties of root systems and algebraic groups. 
Moreover, our technique can be extended to give a new proof of a theorem of Broer's [2, Theorem 1] that characterizes when the restriction mapping from $\operatorname{Mor}_{G}(\mathfrak{g}, V)$ to $\operatorname{Mor}_{W}\left(\mathfrak{t}, V^{T}\right)$ is an isomorphism for a rational $G$-module $V$. In a subsequent paper we hope to give a parabolic analog of Broer's theorem, where $\mathfrak{g}$ is replaced by $\operatorname{Ad} G(\mathfrak{z}+\mathfrak{u}$ ) (the closure of a Dixmier sheet in $\mathfrak{g}$ ), $\mathfrak{t}$ is replaced by $\mathfrak{z}$, and $V^{T}$ is replaced by $V^{L}$.

The rest of this paper is organized as follows. In $\S 2$ we collect several results about hyperplane arrangements in the form we need later; in $\S 3$ we discuss associated fibre bundles over $G / P$; in $\S 4$ we collect some results about the adjoint action of $U$ on $\mathfrak{z}+\mathfrak{u}$; in $\S 5$ we construct $\Gamma$ and prove it's an isomorphism; and in $\S 6$ we explain how the fact that $\Gamma$ is an isomorphism implies the results discussed above.

Finally, we use the following notation throughout this paper. If $X$ and $Y$ are varieties defined over $k$, then $k[X]$ denotes the $k$-algebra of global regular functions on $X$ and $\operatorname{Mor}(X, Y)$ denotes the set of all morphisms from $X$ to $Y$. If a group, $G$, acts on $X$ and $Y$, then $\operatorname{Mor}_{G}(X, Y)$ denotes the set of all $G$-equivariant morphisms from $X$ to $Y$. If $V$ and $W$ are $k$-vector spaces, then $V \otimes W$ and $\operatorname{Hom}(V, W)$ denote $V \otimes_{k} W$ and $\operatorname{Hom}_{k}(V, W)$ respectively. If $V$ and $W$ afford representations of $G$, then $\operatorname{Hom}_{G}(V, W)$ denotes the set of all $G$-equivariant linear transformations from $V$ to $W$.

\section{Hyperplane ARRANGEMENTS}

In this paper a "hyperplane arrangement" is a pair, $(V, \mathcal{A})$, where $V$ is a finite dimensional $k$-vector space and $\mathcal{A}$ is a finite multiset of hyperplanes in $V$. This slight generalization of the usual meaning has no effect on the invariants of arrangements we consider.

Suppose $(V, \mathcal{A})$ is an arrangement with $\operatorname{dim} V=n$. Let $\left\{\mu_{j} \mid 1 \leq j \leq m\right\}$ be a multiset of linear functionals so that $\mathcal{A}=\left\{\operatorname{ker} \mu_{1}, \ldots, \operatorname{ker} \mu_{m}\right\}$. Fix a basis, $\left\{e_{1}, \ldots, e_{n}\right\}$, of $V$ and let $\left\{x_{1}, \ldots, x_{n}\right\}$ be the dual basis of $V^{*}$. Then for $1 \leq i \leq m$ we may write $\mu_{j}=\sum_{i=1}^{n} \xi_{i, j} x_{i}$ where the $\xi_{i, j}$ 's are in $k$. We may identify $k[V]$ with the polynomial ring $k\left[x_{1}, \ldots, x_{n}\right]$ and if $\partial_{i}$ denotes partial differentiation with respect to $x_{i}$, then Der $k[V]$, the $k[V]$-module of $k$-linear derivations of $k[V]$, has basis $\left\{\partial_{1}, \ldots, \partial_{n}\right\}$.

With pointwise operations, $\operatorname{Mor}(V, V)$ is naturally a $k[V]$-module, isomorphic to $k[V] \otimes V$. Suppose $\theta=\sum_{i=1}^{n} f_{i} \partial_{i}$ is in Der $k[V]$. Define $\phi: V \rightarrow V$ by $\phi(v)=$ $\sum_{i=1}^{n} f_{i}(v) e_{i}$. Then it is easily seen that $\phi$ is in $\operatorname{Mor}(V, V)$ and that the mapping $\theta \mapsto \phi$ is an isomorphism of $k[V]$-modules.

Recall that the module of $\mathcal{A}$-derivations, $D(\mathcal{A})$, is the $k[V]$-module of all derivations, $\theta$, of $k[V]$ with the property that $\theta(q)$ is in $k[V] q$ where $q=\prod_{j=1}^{m} \mu_{j}$ is the defining polynomial of $\mathcal{A}$. It's known that $D(\mathcal{A})$ may be characterized as the set of all $\theta$ in Der $k[V]$ with the property that $\theta\left(\mu_{i}\right)$ is in $k[V] \mu_{i}$ for $1 \leq i \leq m[7$, Proposition 4.8]. If $\theta$ and $\phi$ are as in the preceding paragraph, then $\theta$ is in $D(\mathcal{A})$ if and only if $\sum_{i=1}^{n} \xi_{i, j} f_{i}$ is in $k[V] \mu_{j}$ for $1 \leq j \leq m$. On the other hand, if $\phi^{\#}$ is the comorphism of $\phi$, then $\phi^{\#}\left(\mu_{j}\right)=\sum_{i=1}^{n} \xi_{i, j} f_{i}$. Therefore, the image of $D(\mathcal{A})$ under the isomorphism in the preceding paragraph is the set of all morphisms, $\phi: V \rightarrow V$, with the property that $\phi^{\#}\left(\mu_{j}\right)$ is in $k[V] \mu_{j}$ for $1 \leq j \leq m$.

In the rest of this paper, we'll use only morphisms, and not derivations, so from now on, we'll abuse notation and identify $D(\mathcal{A})$ with its image in $\operatorname{Mor}(V, V)$, so $D(\mathcal{A})=\left\{\phi \in \operatorname{Mor}(V, V) \mid \phi^{\#}\left(\mu_{j}\right) \in k[V] \mu_{j}, 1 \leq j \leq m\right\}$. 
Next, recall that the lattice of $\mathcal{A}, L(\mathcal{A})$, is the set of subspaces of $V$ that arise as intersections of hyperplanes in $\mathcal{A}$. Suppose $X$ is in the lattice of $\mathcal{A}$. Then if ker $\mu_{j}$ is in $\mathcal{A}$, either $X \subseteq \operatorname{ker} \mu_{j}$ or $X \cap \operatorname{ker} \mu_{j}$ is a hyperplane in $X$. The "restricted" arrangement, $\left(X, \mathcal{A}^{X}\right)$, is defined to be the arrangement in $X$ consisting of all hyperplanes, $X \cap \operatorname{ker} \mu_{j}$, where $X \nsubseteq \operatorname{ker} \mu_{j}$. Let

$$
X_{\text {reg }}=\left\{v \in X \mid v \in \operatorname{ker} \mu_{j} \text { if and only if } X \subseteq \operatorname{ker} \mu_{j}, 1 \leq j \leq m\right\} .
$$

Then $V$ is the disjoint union of the $X_{\text {reg's as }} X$ runs through $L(\mathcal{A})$. Thus, given $v$ in $V$ we can define $X_{v}$ to be the unique subspace in $L(\mathcal{A})$ with $v$ in $\left(X_{v}\right)_{\text {reg. }}$.

Proposition 2.1. Suppose $\phi: V \rightarrow V$ is a morphism. Then the following are equivalent:

1. $\phi$ is in $D(\mathcal{A})$,

2. $\phi(v) \in X_{v}$ for all $v$ in $V$,

3. $\phi(X) \subseteq X$ for all $X$ in $L(\mathcal{A})$.

Proof. Suppose first that $\phi$ is in $D(\mathcal{A})$. Fix $v$ in $V$. If $X_{v}=X$, then the result is obvious. Suppose $X_{v} \neq X$. Then relabeling if necessary we may assume that $v$ is in ker $\mu_{j}$ if and only if $1 \leq j \leq r$, for some $r$ with $1 \leq r \leq m$. Then $X \subseteq \operatorname{ker} \mu_{j}$ if and only if $1 \leq j \leq r$ and $X_{v}=\bigcap_{j=1}^{r} \operatorname{ker} \mu_{j}$. By assumption, for $1 \leq j \leq r$, $\mu_{j}(v)=0$ implies that $\mu_{j} \phi(v)=0$, so $\phi(v)$ is in $\bigcap_{j=1}^{r} \operatorname{ker} \mu_{j}=X_{v}$.

The second statement clearly implies the third since if $v$ is in $X$, then $X_{v}$ is a subset of $X$.

Finally, suppose $\phi(X) \subseteq X$ for all $X$ in $L(\mathcal{A})$. Then $\phi\left(\operatorname{ker} \mu_{j}\right) \subseteq \operatorname{ker} \mu_{j}$ for $1 \leq j \leq m$. It follows that $\operatorname{ker} \mu_{j}$ is contained in the zero set of $\phi^{\#}\left(\mu_{j}\right)$ and so by the Nullstellensatz, some power of $\phi^{\#}\left(\mu_{j}\right)$ is a multiple of $\mu_{j}$. Since $\mu_{j}$ is irreducible, it must be the case that $\phi^{\#}\left(\mu_{j}\right)$ is a multiple of $\mu_{j}$. Therefore $\phi$ is in $D(\mathcal{A})$.

\section{Fibre Bundles}

In this section, $G$ is an affine algebraic group and $P$ is a closed subgroup of $G$.

Suppose $W$ is a finite dimensional $k$-vector space that affords a rational representation of $P$. Then $P$ acts on $G \times W$ by $p(g, w)=\left(g p^{-1}, p w\right)$ for $p$ in $P, g$ in $G$, and $w$ in $W$. We can form the associated fibre bundle, $G \times{ }^{P} W$, over $G / P$, which as a set is the set of $P$-orbits on $G \times W$. For $g$ in $G$ and $w$ in $W, g * w$ will denote the image of $(g, w)$ in $G \times{ }^{P} W$.

For a non-negative integer, $j$, let $k[W]_{j}$ denote the vector space of all homogeneous, degree $j$ polynomial functions on $W$. Since $P$ acts linearly on $W, k[W]_{j}$ is a $P$-invariant subspace of $k[W]$. The grading, $k[W] \cong \bigoplus_{j \geq 0} k[W]_{j}$, induces a grading, $(k[G] \otimes k[W])^{P} \cong \bigoplus_{j \geq 0}\left(k[G] \otimes k[W]_{j}\right)^{P}$, of $(k[G] \otimes k[W])^{P}$. The natural map from $(k[G] \otimes k[W])^{P}$ to $k\left[G \times{ }^{P} W\right]$ is an isomorphism of $k$-algebras and it's straightforward to check that the image of $\left(k[G] \otimes k[W]_{j}\right)^{P}$ in $k\left[G \times{ }^{P} W\right]$ is

$$
\left\{f \in k\left[G \times{ }^{P} W\right] \mid f(g * \xi w)=\xi^{j} f(g * w) \text { for all } g \in G, w \in W, \xi \in k\right\} .
$$

Denoting this last set by $k\left[G \times{ }^{P} W\right]_{j}$ we see that $k\left[G \times{ }^{P} W\right]$ is graded by $k\left[G \times{ }^{P} W\right]=$ $\bigoplus_{j \geq 0} k\left[G \times{ }^{P} W\right]_{j}$.

Left multiplication defines a regular action of $G$ on $G \times{ }^{P} W$ and so the coordinate ring, $k\left[G \times{ }^{P} W\right]$, affords a representation of $G$. Clearly each $k\left[G \times{ }^{P} W\right]_{j}$ is a $G$-invariant subspace. Moreover, if $f=\sum_{j \geq 0} f_{j}$ is in $k\left[G \times{ }^{P} W\right]^{G}$ with $f_{j}$ 
in $k\left[G \times{ }^{P} W\right]_{j}$, then for fixed $g$ and $w$, and any $\xi$ in $k, f(g * \xi w)$ is a polynomial in $\xi$ with coefficients $f_{j}(g * w)$. Since $f(g * \xi w)=f(1 * \xi w)$, it follows that the coefficients don't depend on $g$ and so each $f_{j}$ is in $k\left[G \times{ }^{P} W\right]^{G}$. Therefore, $k\left[G \times{ }^{P} W\right]^{G} \cong \bigoplus_{j \geq 0} k\left[G \times{ }^{P} W\right]_{j}^{G}$ is a graded $k$-algebra.

Now suppose that $V$ is a finite dimensional $k$-vector space that affords a rational representation of $G$. It's easy to see that with pointwise operations $\operatorname{Mor}_{G}\left(G \times{ }^{P} W, V\right)$ is a $k\left[G \times{ }^{P} W\right]^{G}$-module. Also, $\operatorname{Mor}_{G}\left(G \times{ }^{P} W, V\right)$ is isomorphic to the vector space of all $G$-equivariant linear transformations from $V^{*}$ to $k\left[G \times{ }^{P} W\right]$ since $V$ is an affine space. Thus, $\operatorname{Mor}_{G}\left(G \times{ }^{P} W, V\right) \cong \operatorname{Hom}_{G}\left(V^{*}, k\left[G \times{ }^{P} W\right]\right)$. It's straightforward to check that

$$
\operatorname{Hom}_{G}\left(V^{*}, k\left[G \times{ }^{P} W\right]\right) \cong \bigoplus_{j \geq 0} \operatorname{Hom}_{G}\left(V^{*}, k\left[G \times{ }^{P} W\right]_{j}\right)
$$

and that the image of $\operatorname{Hom}_{G}\left(V^{*}, k\left[G \times{ }^{P} W\right]_{j}\right)$ in $\operatorname{Mor}_{G}\left(G \times{ }^{P} W, V\right)$ is

$$
\left\{\phi \in \operatorname{Mor}_{G}\left(G \times{ }^{P} W, V\right) \mid \phi(g * \xi w)=\xi^{j} \phi(g * w) \text { for all } g \in G, w \in W, \xi \in k\right\} \text {. }
$$

Thus, if we define $\operatorname{Mor}_{G}\left(G \times{ }^{P} W, V\right)_{j}$ to be this last vector space, it follows that $\operatorname{Mor}_{G}\left(G \times{ }^{P} W, V\right) \cong \bigoplus_{j \geq 0} \operatorname{Mor}_{G}\left(G \times{ }^{P} W, V\right)_{j}$. Moreover, it's easy to see that in fact $\operatorname{Mor}_{G}\left(G \times{ }^{P} W, V\right)$ is a graded $k\left[G \times{ }^{P} W\right]^{G}$-module.

Similarly, again since $P$ acts linearly on $W, k[W]^{P} \cong \bigoplus_{j \geq 0} k[W]_{j}^{P}$ and if

$$
\operatorname{Mor}_{P}(W, V)_{j}=\left\{\psi \in \operatorname{Mor}_{P}(W, V) \mid \psi(\xi w)=\xi^{j} w \text { for all } w \in W, \xi \in k\right\},
$$

then $\operatorname{Mor}_{P}(W, V) \cong \bigoplus_{j \geq 0} \operatorname{Mor}_{P}(W, V)_{j}$ and therefore $\operatorname{Mor}_{P}(W, V)$ is a graded $k[W]^{P}$-module.

Let $\tau: W \rightarrow G \times{ }^{P} W$ be the "inclusion" of the fibre over $P$ defined by $\tau(w)=1 * w$ for $w$ in $W$, so $\tau$ is a morphism. Suppose $\phi$ is $\operatorname{in} \operatorname{Mor}_{G}\left(G \times{ }^{P} W, V\right)$. Then, for $p$ in $P$ and $w$ in $W$,

$$
p \phi \tau(w)=\phi(p * w)=\phi(1 * p w)=\phi \tau(p w),
$$

so $\phi \tau$ is in $\operatorname{Mor}_{P}(W, V)$, the $k[W]^{P}$-module of all $P$-equivariant morphisms from $W$ to $V$. We need the following version of Frobenius reciprocity.

Proposition 3.1. The mapping $\phi \mapsto \phi \tau$ defines an isomorphism of graded vector spaces between $\operatorname{Mor}_{G}\left(G \times{ }^{P} W, V\right)$ and $\operatorname{Mor}_{P}(W, V)$.

Proof. First, suppose $\phi$ is in $\operatorname{Mor}_{G}\left(G \times{ }^{P} W, V\right)_{j}$. Then for $w$ in $W$ and $\xi$ in $k$,

$$
\phi \tau(\xi w)=\phi(1 * \xi x)=\xi^{j} \phi(1 * w)=\xi^{j} \phi \tau(w),
$$

so $\phi \tau$ is in $\operatorname{Mor}_{P}(W, V)_{j}$.

It's straightforward to check that the mapping is a homomorphism of graded vector spaces. To show that it's injective, suppose $\phi_{1}$ and $\phi_{2}$ are in $\operatorname{Mor}_{G}\left(G \times{ }^{P} W, V\right)$ and $\phi_{1} \tau=\phi_{2} \tau$. Then for $g$ in $G$ and $w$ in $W$,

$$
\begin{aligned}
\phi_{1}(g * w) & =\phi_{1}(1 * w) \\
& =\phi_{1} \tau(w) \\
& =\phi_{2} \tau(w) \\
& =\phi_{2}(g * w),
\end{aligned}
$$

so $\phi_{1}=\phi_{2}$. 
To show the mapping is surjective, suppose $\widetilde{\phi}$ is in $\operatorname{Mor}_{P}(W, V)$. We define $\phi: G \times{ }^{P} W \rightarrow V$ by $\phi(g * w)=g \widetilde{\phi}(w)$. Then $\phi$ is easily seen to be a well-defined morphism and clearly $\phi \tau=\widetilde{\phi}$. If $g_{1}$ is in $G$, then

$$
\begin{aligned}
\phi\left(g_{1}(g * w)\right) & =\phi\left(g_{1} g * w\right) \\
& =g_{1} g \widetilde{\phi}(w) \\
& =g_{1} \phi(g * w),
\end{aligned}
$$

so $\phi$ is $G$-equivariant. This completes the proof of the proposition.

Corollary 3.2. The mapping $f \mapsto f \tau$ defines an isomorphism of graded $k$-algebras between $k\left[G \times{ }^{P} W\right]^{G}$ and $k[W]^{P}$.

Proof. This is just the preceding proposition when $V=k$ with the trivial action of $G$.

Notice that if $\phi$ is in $\operatorname{Mor}_{G}\left(G \times{ }^{P} W, V\right)$ and $f$ is in $k\left[G \times{ }^{P} W\right]^{G}$, then $(f \phi) \circ \tau=$ $(f \circ \tau)(\phi \circ \tau)$, so the isomorphism above intertwines the $k\left[G \times{ }^{P} W\right]^{G}$-module structure on $\operatorname{Mor}_{G}\left(G \times{ }^{P} W, V\right)$ and the $k[W]^{P}$-module structure on $\operatorname{Mor}_{P}(W, V)$.

Now suppose that $W_{1}$ is a subspace of $W$ with the property that $p w-w$ is in $W_{1}$ for all $p$ in $P$ and $w$ in $W$. Notice that $W_{1}$ is a $P$-stable subspace of $W$ and that $P$ acts trivially on $W / W_{1}$. Let $W_{0}$ be a complement to $W_{1}$ in $W$. Then we may identify $k[W]$ and $k\left[W_{0}\right] \otimes k\left[W_{1}\right]$. If $k\left[W_{0}\right]$ is given the trivial $P$-action and $P$ acts diagonally on $k\left[W_{0}\right] \otimes k\left[W_{1}\right]$, then this identification is $P$-equivariant.

Let $\theta: G \times{ }^{P} W \rightarrow W_{0}$ by $\theta\left(g *\left(w_{0}+w_{1}\right)\right)=w_{0}$. Notice that $\theta$ is well-defined since $P$ acts trivially on $W / W_{1}$. The comorphism, $\theta^{\#}: k\left[W_{0}\right] \rightarrow k\left[G \times{ }^{P} W\right]$ defines the structure of a graded $k\left[W_{0}\right]$-module on $k\left[G \times{ }^{P} W\right]$.

Since $P$ acts trivially on $k\left[W_{0}\right]$ in the decomposition $k[W] \cong k\left[W_{0}\right] \otimes k\left[W_{1}\right]$ we have $G$-equivariant isomorphisms of graded $k\left[W_{0}\right]$-modules,

$$
\begin{aligned}
k\left[G \times{ }^{P} W\right] & \cong\left(k[G] \otimes k\left[W_{0}\right] \otimes k\left[W_{1}\right]\right)^{P} \\
& \cong k\left[W_{0}\right] \otimes\left(k[G] \otimes k\left[W_{1}\right]\right)^{P} \\
& \cong k\left[W_{0}\right] \otimes k\left[G \times{ }^{P} W_{1}\right],
\end{aligned}
$$

where $G$ acts trivially on $k\left[W_{0}\right]$ and diagonally on $k\left[W_{0}\right] \otimes k\left[G \times{ }^{P} W_{1}\right]$.

More generally, using the isomorphism in the last paragraph, we have isomorphisms of graded $k\left[W_{0}\right]$-modules,

$$
\begin{aligned}
\operatorname{Mor}_{G}\left(G \times{ }^{P} W, V\right) & \cong \operatorname{Hom}_{G}\left(V^{*}, k\left[G \times{ }^{P} W\right]\right) \\
& \cong\left(V \otimes k\left[W_{0}\right] \otimes k\left[G \times{ }^{P} W_{1}\right]\right)^{G} \\
& \cong k\left[W_{0}\right] \otimes\left(V \otimes k\left[G \times{ }^{P} W_{1}\right]\right)^{P} \\
& \cong k\left[W_{0}\right] \otimes \operatorname{Hom}_{G}\left(V^{*}, k\left[G \times{ }^{P} W_{1}\right]\right) .
\end{aligned}
$$

This proves the next lemma.

Lemma 3.3. There is an isomorphism graded $k\left[W_{0}\right]$-modules,

$$
\operatorname{Mor}_{G}\left(G \times{ }^{P} W, V\right) \cong k\left[W_{0}\right] \otimes \operatorname{Hom}_{G}\left(V^{*}, k\left[G \times{ }^{P} W_{1}\right]\right) .
$$

Proposition 3.4. The comorphism, $\theta^{\#}: k\left[W_{0}\right] \rightarrow k\left[G \times{ }^{P} W\right]$, is an injective homomorphism of graded $k$-algebras. Moreover, if $P W_{0}$ is a dense subvariety of $W$, 
then $\theta^{\#}\left(k\left[W_{0}\right]\right)=k\left[G \times{ }^{P} W\right]^{G}$. Thus, if $P W_{0}$ is dense in $W$, then $k\left[W_{0}\right]$ and $k\left[G \times{ }^{P} W\right]^{G}$ are isomorphic, graded k-algebras.

Proof. It's easy to see that $\theta^{\#}$ is an injective homomorphism of graded $k$-algebras and that $\theta^{\#}\left(k\left[W_{0}\right]\right) \subseteq k\left[G \times{ }^{P} W\right]^{G}$. Now suppose that $P W_{0}$ is dense in $W$. For $f$ in $k\left[W_{0}\right]^{G}$, define $f_{1}: W_{0} \rightarrow k$ by $f_{1}\left(w_{0}\right)=f\left(1 * w_{0}\right)$, so $f_{1}$ is in $k\left[W_{0}\right]$. Suppose $g$ is in $G, p$ is in $P$, and $w_{0}$ is in $W_{0}$. Then,

$$
\begin{aligned}
f_{1} \theta\left(g * p w_{0}\right) & =f_{1} \theta\left(g p * w_{0}\right) \\
& =f_{1}\left(w_{0}\right) \\
& =f\left(1 * w_{0}\right) \\
& =f\left(g p * w_{0}\right) \\
& =f\left(g * p w_{0}\right) .
\end{aligned}
$$

Therefore $f_{1} \theta$ and $f$ agree on

$$
G * P W_{0}=\left\{g * p w_{0} \mid g \in G, p \in P, w_{0} \in W_{0}\right\} .
$$

Since $P W_{0}$ is dense in $W, G * P W_{0}$ is dense in $G \times{ }^{P} W$, and so $f_{1} \theta=f$. Therefore $\theta^{\#}\left(f_{1}\right)=f$ and hence $k\left[G \times{ }^{P} W\right]^{G} \subseteq \theta^{\#}\left(k\left[W_{0}\right]\right)$. This completes the proof of the proposition.

\section{The ACtion of $U$ ON $\mathfrak{z}+\mathfrak{u}$}

For the rest of this paper, we return to the notation of the introduction: $G$ is a connected, reductive, algebraic group; $T$ is a maximal torus in $G ; P$ is a parabolic subgroup of $G$ with Levi decomposition $P=L U$; and $\mathfrak{z}$ is the center of $\mathfrak{l}$. Also, let $\Phi$ denote the root system of $(\mathfrak{g}, \mathfrak{t})$ and let $\Phi_{\mathfrak{u}}$ be the subset of $\Phi$ consisting of the weights of $\mathfrak{t}$ on $\mathfrak{u}$.

Lemma 4.1. Suppose $l$ is a semisimple element in $\mathfrak{l}$. Define $a_{l}: U \times Z_{\mathfrak{u}}(l) \rightarrow l+\mathfrak{u}$ by $a_{l}(u, z)=\operatorname{Ad} u(l+z)$. Then $a_{l}$ is surjective with fibres isomorphic to $Z_{U}(l)$.

Proof. To show that $a_{l}$ is surjective, suppose $n$ is in $\mathfrak{u}$. Let $l+n=(l+n)_{s}+(l+n)_{n}$ be the Jordan decomposition on $l+n$. Then Borho [1, §2] has shown that there is a $u$ in $U$ with $\operatorname{Ad} u(l)=(l+n)_{s}$. Set $z=\operatorname{Ad} u^{-1}\left((l+n)_{n}\right)$, so $\operatorname{Ad} u(z)=(l+n)_{n}$. Then clearly $\operatorname{Ad} u(l+z)=l+n$. Moreover, $[\operatorname{Ad} u(l), \operatorname{Ad} u(z)]=\left[(l+n)_{s},(l+n)_{n}\right]=0$. Thus, $z$ is in $Z_{\mathfrak{u}}(l)$ and $a_{l}(u, z)=l+n$.

Suppose $n$ is in $\mathfrak{u}$ and consider $F=a_{l}^{-1}(l+n)$. Fix $(u, z)$ in $F$. Since $\operatorname{Ad} u(l+z)$ has Jordan decomposition $\operatorname{Ad} u(l+z)=\operatorname{Ad} u(l)+\operatorname{Ad} u(z)$, if $\left(u_{1}, z_{1}\right)$ is in $U \times Z_{\mathfrak{u}}(l)$, then $\left(u_{1}, z_{1}\right)$ is in $F$ if and only if $\operatorname{Ad} u_{1}(l)=\operatorname{Ad} u(l)$ and $\operatorname{Ad} u_{1}\left(z_{1}\right)=\operatorname{Ad} u(z)$. Thus $\rho: F \rightarrow u Z_{U}(l)$ by $\rho\left(u_{1}, z_{1}\right)=u_{1}$ is a well-defined morphism. If $\rho\left(u_{1}, z_{1}\right)=$ $\rho\left(u_{2}, z_{2}\right)$, then $u_{1}=u_{2}$ and so $\operatorname{Ad} u_{1}\left(z_{1}\right)=\operatorname{Ad} u_{1}\left(z_{2}\right)$, so $z_{1}=z_{2}$ and hence $\rho$ is injective. If $v$ is in $Z_{U}(l)$, set $u_{1}=u v$ and $z_{1}=\operatorname{Ad} v^{-1}(l+z)-l$. It's straightforward to check that $\left(u_{1}, z_{1}\right)$ is in $F$ and so $\rho$ is surjective. Finally, $\rho$ is an isomorphism by Zariski's Main Theorem.

Corollary 4.2. The P-saturation of $\mathfrak{z}_{\text {reg }}, \operatorname{Ad} P\left(\mathfrak{z}_{\text {reg }}\right)$, is $\mathfrak{z}_{\text {reg }}+\mathfrak{u}$, so $\operatorname{Ad} P\left(\mathfrak{z}_{\text {reg }}\right)$ is a dense open subvariety of $\mathfrak{z}+\mathfrak{u}$.

Proof. Recall that $\left(\mathfrak{z}, \mathcal{A}^{\mathfrak{z}}\right)$ is an arrangement in $\mathfrak{z}$, so $\mathfrak{z}$ reg is defined as in $\S 2$. It's easy to see that $\mathfrak{z}_{\text {reg }}=\left\{t \in \mathfrak{t} \mid \alpha(t) \neq 0\right.$ for all $\left.\alpha \in \Phi_{\mathfrak{u}}\right\}$ and hence that $t$ is in $\mathfrak{z}_{\text {reg }}$ if and only if $Z_{\mathfrak{u}}(t)=0$. If $t$ is in $\mathfrak{z}$ reg, then it follows from Lemma 4.1 that $\operatorname{Ad} P(t)=t+\mathfrak{u}$, so $\operatorname{Ad} P\left(\mathfrak{z}_{\text {reg }}\right)=\mathfrak{z}$ reg $+\mathfrak{u}$. 
For $\alpha$ in $\Phi$ let $\mathfrak{g}_{\alpha}$ denote the $\alpha$-weight space in $\mathfrak{g}$ and fix a non-zero root vector, $e_{\alpha}$ in $\mathfrak{g}_{\alpha}$. Also, let $U_{\alpha}$ be the corresponding root subgroup in $G$ and suppose $x_{\alpha}: k \rightarrow U_{\alpha}$ is a fixed isomorphism from $k$ to $U_{\alpha}$ satisfying $\operatorname{Ad} x_{\alpha}(\xi)=\exp \left(\operatorname{ad}\left(\xi e_{\alpha}\right)\right)$ for $\xi$ in $k$, where ad is the adjoint representation of $\mathfrak{g}$.

For $\alpha$ and $\beta$ in $\Phi_{\mathfrak{u}}$, define $\alpha \sim \beta$ if $\left.\operatorname{ker} \alpha\right|_{\mathfrak{z}}=\left.\operatorname{ker} \beta\right|_{\mathfrak{z}}$. Clearly this is an equivalence relation on $\Phi_{\mathfrak{u}}$. It's also clear that for $\alpha$ and $\beta$ in $\Phi_{\mathfrak{u}}, \alpha \sim \beta$ if and only if $\left.\alpha\right|_{\mathfrak{z}}$ is a scalar multiple of $\left.\beta\right|_{\mathfrak{z}}$. Let $\Psi_{1}, \ldots, \Psi_{s}$ be the equivalence classes for this relation on $\Phi_{\mathfrak{u}}$.

Notice that each $\Psi_{i}$ is a closed set of roots (that is, if $\alpha$ and $\beta$ are in $\Psi_{i}$ and $\alpha+\beta$ is a root, then $\alpha+\beta$ is in $\Psi_{i}$ ). It follows that if we define $\mathfrak{u}_{i}$ to be the span of the root vectors $e_{\alpha}$ with $\alpha$ in $\Psi_{i}$ and $U_{i}$ to be the subgroup of $U$ generated by the root subgroups, $U_{\alpha}$, where $\alpha$ is in $\Psi_{i}$, then $\mathfrak{u}_{i}$ is a subalgebra of $\mathfrak{u}, U_{i}$ is a subgroup of $U$ isomorphic to the product $\prod_{\alpha \in \Psi_{i}} U_{\alpha}$ (the product can be taken in any order), and $\operatorname{Lie}\left(U_{i}\right)=\mathfrak{u}_{i}$. Moreover, if $U^{i}=\prod_{\beta \in \Phi_{\mathfrak{u}} \backslash \Psi_{i}} U_{\beta}$ (the product taken in some fixed order), then every element in $U$ has a unique factorization $u=u^{i} u_{i}$ with $u^{i}$ in $U^{i}$ and $u_{i}$ in $U_{i}$.

For $1 \leq i \leq s$, let $H_{i}$ denote $\left.\operatorname{ker} \alpha\right|_{\mathfrak{z}}$ for $\alpha$ in $\Psi_{i}$, so $H_{1}, \ldots, H_{s}$ are the distinct hyperplanes in $\mathcal{A}^{\mathfrak{z}}$. Set $\mathfrak{z}^{i}=\left\{t \in \mathfrak{z} \mid \beta(t) \neq 0\right.$ for all $\left.\beta \in \Phi_{\mathfrak{u}} \backslash \Psi_{i}\right\}=\mathfrak{z} \backslash \bigcup_{i \neq j} H_{j}$. Then $\mathfrak{z}^{i}$ is an open subvariety of $\mathfrak{z}$ containing $\mathfrak{z}_{\text {reg }}$. Finally, define $a: U \times(\mathfrak{z}+\mathfrak{u}) \rightarrow$ $\mathfrak{z}+\mathfrak{u}$ by $a(u, t+n)=\operatorname{Ad} u(t+n)$ and let $a_{i}$ denote the restriction of $a$ to $U^{i} \times\left(\mathfrak{z}^{i}+\mathfrak{u}_{i}\right)$.

Proposition 4.3. For $1 \leq i \leq s$, the morphism $a_{i}: U^{i} \times\left(\mathfrak{z}^{i}+\mathfrak{u}_{i}\right) \rightarrow \mathfrak{z}+\mathfrak{u}$ determines an isomorphism between $U^{i} \times\left(\mathfrak{z}^{i}+\mathfrak{u}_{i}\right)$ and $\mathfrak{z}^{i}+\mathfrak{u}$.

Proof. We show that $a_{i}$ is injective with image equal to $\mathfrak{z}^{i}+\mathfrak{u}$. Then by restricting the range we obtain a bijective, birational morphism of normal varieties between $U^{i} \times\left(\mathfrak{z}^{i}+\mathfrak{u}_{i}\right)$ and $\mathfrak{z}^{i}+\mathfrak{u}$ which must be an isomorphism by Zariski's Main Theorem.

To show that $a_{i}$ is injective, suppose $\operatorname{Ad} u(t+n)=\operatorname{Ad} u_{1}\left(t_{1}+n_{1}\right)$ for $u, u_{1}$ in $U^{i}, t, t_{1}$ in $\mathfrak{z}^{i}$, and $n, n_{1}$ in $\mathfrak{u}_{i}$. Then clearly $t=t_{1}$ and $\operatorname{Ad} u_{1}^{-1} u(t+n)=t+n_{1}$. Just suppose $u_{1}{ }^{-1} u$ is not in $U_{i}$ and choose $\beta$ in $\Phi_{\mathfrak{u}}$ with $\beta$ not in $\Psi_{i}$, so that the height of $\beta$ is minimal and such that $u_{1}^{-1} u=x_{\beta}(c) u^{\prime}$ for some $u^{\prime}$ in $U$, with $c \neq 0$. We show that $\operatorname{Ad} x_{\beta}(c) u^{\prime}(t+n)=t-c \beta(t)+\sum_{\alpha \neq \beta} r_{\alpha} e_{\alpha}$. First, if $e_{\alpha}$ occurs with a non-zero coefficient in $\operatorname{Ad} u^{\prime}(t)$, then $\alpha=\gamma_{1}+\cdots+\gamma_{m}$ with $\gamma_{i} \neq \beta$ for $1 \leq i \leq m$. If some $\gamma_{j}$ are not in $\Psi_{i}$, then either $m=1$ and $\alpha \neq \beta$, or $m>1$ in which case the height of $\alpha$ is strictly greater than the height of $\beta$, so again $\alpha \neq \beta$. If every $\gamma_{j}$ is in $\Psi_{i}$, then so is their sum, so again $\alpha \neq \beta$. It follows that $\operatorname{Ad} u^{\prime}(t)=t+\sum_{\alpha \neq \beta} s_{\alpha} e_{\alpha}$ for some $s_{\alpha}$ 's in $k$. Thus $\operatorname{Ad} x_{\beta}(c) u^{\prime}(t)=t-c \beta(t)+\sum_{\alpha \neq \beta} s_{\alpha}^{\prime} e_{\alpha}$ where the $s_{\alpha}^{\prime}$ 's are in $k$. Next, if $e_{\alpha}$ occurs with a non-zero coefficient in $\operatorname{Ad} x_{\beta}(c) u^{\prime}(n)$, then again, either $\alpha$ is in $\Psi_{i}$ in which case $\alpha \neq \beta$, or $\alpha$ is not in $\Psi_{i}$ in which case the height of $\alpha$ is strictly greater than the height of $\beta$, so $\alpha \neq \beta$ in this case either. Thus, $e_{\beta}$ does not appear in $\operatorname{Ad} x_{\beta}(c) u^{\prime}(n)$. It follows that $\operatorname{Ad} x_{\beta}(c) u^{\prime}(t+n)=t-c \beta(t)+\sum_{\alpha \neq \beta} r_{\alpha} e_{\alpha}$ as desired. Since $\beta \neq \alpha$, it must be that $c \beta(t)=0$ and since $t$ is in $\mathfrak{z}^{i}, \beta(t) \neq 0$, so $c=0$, a contradiction. It follows that $u_{1}{ }^{-1} u$ is in $U_{i}$ and so it follows from the factorization $U=U^{i} U_{i}$ that $u=u_{1}$. Therefore $n=n_{1}$ also and so $a_{i}$ is injective.

To show that the image of $a_{i}$ is $\mathfrak{z}^{i}+\mathfrak{u}$, suppose that $t$ is in $\mathfrak{z}^{i}$. If $t$ is in $H_{i}$, then $Z_{\mathfrak{u}}(t)=\mathfrak{u}_{i}$ since $t$ is not in $H_{j}$ for $j \neq i$, so by Lemma 4.1 given $n$ in $\mathfrak{u}$, there is a $u$ in $U$ and an $n_{1}$ in $\mathfrak{u}_{i}$ so that $\operatorname{Ad} u\left(t+n_{1}\right)=t+n$. Write $u=u^{i} u_{i}$ with $u^{i}$ in $U^{i}$ and $u_{i}$ in $U_{i}$. Then $\operatorname{Ad} u_{i}\left(t+n_{1}\right)$ is in $t+\mathfrak{u}_{i}$ (recall that $\Psi_{i}$ is a closed set of roots), so say $\operatorname{Ad} u_{i}\left(t+n_{1}\right)=t+n_{1}^{\prime}$ where $n_{1}^{\prime}$ is in $\mathfrak{u}_{i}$. It follows that $t+n=\operatorname{Ad} u^{i}\left(t+n_{1}^{\prime}\right)$ 
is in the image of $a_{i}$. If $t$ is not in $H_{i}$, then $t$ is in $z_{\text {reg }}$ and so again by Lemma 4.1, given $n$ in $\mathfrak{u}$ there is a $u$ in $U$ with $\operatorname{Ad} u(t)=t+n$. Factor $u=u^{i} u_{i}$ as above. Then $\operatorname{Ad} u_{i}(t)$ is in $t+\mathfrak{u}_{i}$, say $\operatorname{Ad} u_{i}(t)=t+n_{1}$. Then $t+n=\operatorname{Ad} u^{i}\left(t+n_{1}\right)$, and so $t+n$ is in the image of $a_{i}$.

For $1 \leq i \leq s$, fix a root, say $\alpha_{i}$, in $\Psi_{i}$. This set of representatives will remain fixed for the rest of this paper.

Proposition 4.4. Suppose $1 \leq i \leq s$ and $u$ is in $U_{i}$. Then there is an element, $n_{1}$, in $\mathfrak{u}_{i}$, so that for $t$ in $\mathfrak{z}, \operatorname{Ad} u(t)=t+\alpha_{i}(t) n_{1}$.

Proof. We can write $u=x_{\beta_{n}}\left(c_{n}\right) \cdots x_{\beta_{1}}\left(c_{1}\right)$ where the $c_{j}$ 's are in $k$ and $\beta_{1}, \ldots, \beta_{n}$ are in $\Psi_{i}$. We prove the result using induction on $n$. If $n=1$, then $\operatorname{Ad} x_{\beta_{1}}\left(c_{1}\right)(t)=$ $t-\beta_{1}(t) c_{1} e_{\beta_{1}}$. Since $\beta_{1}$ is in $\Psi_{i}$, there is a rational number, $r$, so that $\left.\beta\right|_{\mathfrak{z}}=\left.r \alpha\right|_{\mathfrak{z}}$. Now we can take $n_{1}=-r c_{1} e_{\beta_{1}}$.

Suppose that $n>1, u=x_{\beta_{n}}\left(c_{n}\right) \cdots x_{\beta_{1}}\left(c_{1}\right)$, and

$$
\operatorname{Ad}\left(x_{\beta_{n-1}}\left(c_{n-1}\right) \cdots x_{\beta_{1}}\left(c_{1}\right)\right)(t)=t+\alpha_{i}(t) n_{1}^{\prime}
$$

for some $n_{1}^{\prime}$ in $\mathfrak{u}_{i}$ and all $t$ in $\mathfrak{z}$. Then

$$
\begin{aligned}
\operatorname{Ad} u(t) & =\operatorname{Ad} x_{\beta_{n}}\left(c_{n}\right)\left(t+\alpha_{i}(t) n_{1}^{\prime}\right) \\
& =t-\beta_{n}(t) c_{n} e_{\beta_{n}}+\alpha_{i}(t) \operatorname{Ad} x_{\beta_{n}}\left(c_{n}\right) n_{1}^{\prime} .
\end{aligned}
$$

Now $\beta_{n}$ is in $\Psi_{i}$, so $\left.\beta_{n}\right|_{\mathfrak{z}}=\left.r \alpha\right|_{\mathfrak{z}}$ for some rational number, $r$, and $\operatorname{Ad} x_{\beta_{n}}\left(c_{n}\right) n_{1}^{\prime}=n_{1}^{\prime \prime}$ for some $n_{1}^{\prime \prime}$ in $\mathfrak{u}_{i}$. The result follows by taking $n_{1}=-c_{n} r e_{\beta_{n}}+n_{1}^{\prime \prime}$.

\section{THE ISOMORPHISM}

Let $\Phi_{\mathfrak{l}}$ denote the root system of $(\mathfrak{l}, \mathfrak{t})$, so $\Phi$ is the disjoint union of $\Phi_{\mathfrak{l}}, \Phi_{\mathfrak{u}}$, and $-\Phi_{\mathfrak{u}}$. Recall that $\mathfrak{z} \subseteq \mathfrak{t}$ and that $\mathfrak{z}=\bigcap_{\alpha \in \Phi_{\mathfrak{l}}}$ ker $\left.\alpha\right|_{\mathfrak{z}}$. Also, for $\alpha$ in $\Phi, \mathfrak{z} \subseteq \operatorname{ker} \alpha$ if and only if $\alpha \in \Phi_{\mathfrak{l}}$. Thus the hyperplanes, $\left.\operatorname{ker} \alpha\right|_{\mathfrak{z}}$, for $\alpha$ in $\Phi_{\mathfrak{u}}$ determine a hyperplane arrangement in $\mathfrak{z},\left(\mathfrak{z}, \mathcal{A}^{\mathfrak{z}}\right)$. As in $\S 2$ we consider elements in $D\left(\mathcal{A}^{\mathfrak{z}}\right)$ as morphisms from $\mathfrak{z}$ to itself satisfying the equivalent conditions of Proposition 2.1.

For $t$ in $\mathfrak{z}$, set $\Psi_{t}=\left\{\alpha \in \Phi_{\mathfrak{u}} \mid \alpha(t)=0\right\}$. Then, with the notation of $\S 2$, $X_{t}=\left.\bigcap_{\alpha \in \Psi_{t}} \operatorname{ker} \alpha\right|_{\mathfrak{z}}$. Recall that for $\alpha$ in $\Phi$, the root subgroup of $G$ corresponding to $\alpha$ is $U_{\alpha}$. Then $Z_{U}(t)=\prod_{\alpha \in \Psi_{t}} U_{\alpha}$ and $Z_{P}(t)=L Z_{U}(t)$.

Let $\sigma: \mathfrak{z} \rightarrow G \times{ }^{P}(\mathfrak{z}+\mathfrak{u})$ by $\sigma(t)=1 * t$, so $\sigma$ is a morphism. Suppose that $\phi: G \times{ }^{P}(\mathfrak{z}+\mathfrak{u}) \rightarrow \mathfrak{g}$ is a $G$-equivariant morphism. Then $\phi \sigma: \mathfrak{z} \rightarrow \mathfrak{g}$ is a morphism and since $\phi$ is $G$-equivariant and the stabilizer in $G$ of $\sigma(t)$ is $Z_{P}(t)$, it follows that $\phi \sigma(t)$ is in $\mathfrak{g}^{Z_{P}(t)}$ for $t$ in $\mathfrak{z}$. Now $\mathfrak{g}^{Z_{P}(t)} \subseteq \mathfrak{g}^{L}=\mathfrak{z}$ and so

$$
\mathfrak{g}^{Z_{P}(t)}=\left\{t_{1} \in \mathfrak{z} \mid \alpha\left(t_{1}\right)=0 \text { for all } \alpha \in \Psi_{t}\right\}=X_{t} .
$$

Therefore, $\phi \sigma(\mathfrak{z}) \subseteq \mathfrak{z}$ and for $t$ in $\mathfrak{z}, \phi \sigma(t) \in X_{t}$, so by restricting the range of $\phi \sigma$ to $\mathfrak{z}$ we obtain a morphism, $\Lambda(\phi)$, in $D\left(\mathcal{A}^{\mathfrak{z}}\right)$.

The rest of this section is devoted to the proof of the following theorem.

Theorem 5.1. The mapping $\Lambda: \operatorname{Mor}_{G}\left(G \times{ }^{P}(\mathfrak{z}+\mathfrak{u}), \mathfrak{g}\right) \rightarrow D\left(\mathcal{A}^{\mathfrak{z}}\right)$ is an isomorphism of graded $k[\mathfrak{z}]$-modules.

It's well-known that $P$ acts trivially on $(\mathfrak{z}+\mathfrak{u}) / \mathfrak{u}$. Therefore, we can apply the results in $\S 3$ with $W=\mathfrak{z}+\mathfrak{u}, W_{0}=\mathfrak{z}$, and $W_{1}=\mathfrak{u}$, and conclude that $\operatorname{Mor}_{G}\left(G \times{ }^{P}(\mathfrak{z}+\mathfrak{u}), \mathfrak{g}\right)$ has the structure of a graded $k[\mathfrak{z}]$-module via the comorphism 
of $\theta: G \times{ }^{P}(\mathfrak{z}+\mathfrak{u}) \rightarrow \mathfrak{z}$. Moreover, by Corollary 4.2, $\operatorname{Ad} P(\mathfrak{z})$ is dense in $\mathfrak{z}+\mathfrak{u}$, and so $k[\mathfrak{z}]$ and $k\left[G \times{ }^{P}(\mathfrak{z}+\mathfrak{u})\right]^{G}$ are isomorphic graded $k$-algebras by Proposition 3.4.

It's straightforward to check that $\Lambda$ is a homomorphism of $k[\mathfrak{z}]$-modules. Moreover, if $\phi$ is in $\operatorname{Mor}_{G}\left(G \times{ }^{P}(\mathfrak{z}+\mathfrak{u}), \mathfrak{g}\right)_{j}$ for some non-negative integer, $j$, then

$$
\Lambda(\phi(\xi t))=\phi(1 * \xi t)=\xi^{j} \Lambda(\phi)(t)
$$

for $t$ in $\mathfrak{z}$ and $\xi$ in $k$, so $\Lambda(\phi)$ is in $D\left(\mathcal{A}^{\mathfrak{z}}\right)_{j}$. Thus $\Lambda$ is a homomorphism of graded $k[\mathfrak{z}]$-modules.

To show that $\Lambda$ is injective, suppose $\phi_{1}$ and $\phi_{2}$ are in $\operatorname{Mor}_{G}\left(G \times{ }^{P}(\mathfrak{z}+\mathfrak{u}), \mathfrak{g}\right)$ with $\Lambda\left(\phi_{1}\right)=\Lambda\left(\phi_{2}\right)$. Define $G *\left(\mathfrak{z}_{\text {reg }}+\mathfrak{u}\right)=\left\{g *(t+n) \mid g \in G, t \in \mathfrak{z}_{\text {reg }}, n \in \mathfrak{u}\right\}$. Then if $g *(t+n)$ is in $G *\left(\mathfrak{z}_{\text {reg }}+\mathfrak{u}\right)$, by Lemma 4.1 there is a $u$ in $U$ with $\operatorname{Ad} u(t)=t+n$ and so

$$
\begin{aligned}
\phi_{1}(g *(t+n)) & =\phi_{1}(g u * t) \\
& =\phi_{1}(1 * t) \\
& =\phi_{1} \sigma(t) \\
& =\phi_{2} \sigma(t) \\
& =\phi_{2}(g u * t) \\
& =\phi_{2}(g *(t+n)) .
\end{aligned}
$$

Therefore, $\phi_{1}$ and $\phi_{2}$ agree on $G *\left(\mathfrak{z}_{\text {reg }}+\mathfrak{u}\right)$. Since $\mathfrak{z}_{\text {reg }}+\mathfrak{u}$ is a dense subvariety of $\mathfrak{z}+\mathfrak{u}, G *\left(\mathfrak{z}_{\mathrm{reg}}+\mathfrak{u}\right)$ is a dense subvariety of $G \times{ }^{P}(\mathfrak{z}+\mathfrak{u})$ and so $\phi_{1}=\phi_{2}$.

To show that $\Lambda$ is surjective we proceed as follows. Suppose $\eta$ is in $D\left(\mathcal{A}^{\mathfrak{z}}\right)$. We first construct a $P$-equivariant morphism, $\widetilde{\phi}: \mathfrak{z}+\mathfrak{u} \rightarrow \mathfrak{z}+\mathfrak{u}$ with $\left.\widetilde{\phi}\right|_{\mathfrak{z}}=\eta$. Extending the range of $\widetilde{\phi}$ to all of $\mathfrak{g}$ we can apply Proposition 3.1 and obtain a $G$-equivariant morphism, $\phi: G \times{ }^{P}(\mathfrak{z}+\mathfrak{u}) \rightarrow \mathfrak{g}$, with $\phi(1 *(t+n))=\widetilde{\phi}(t+n)$ for all $t+n$ in $\mathfrak{z}+\mathfrak{u}$. Then clearly $\Lambda(\phi)=\eta$ and so $\Lambda$ is surjective.

Now, to complete the proof of Theorem 5.1 it remains to construct a morphism, $\widetilde{\phi}$, in $\operatorname{Mor}_{P}(\mathfrak{z}+\mathfrak{u}, \mathfrak{z}+\mathfrak{u})$, given a morphism, $\eta$, in $D\left(\mathcal{A}^{\mathfrak{z}}\right)$. So for the rest of this section, fix $\eta$ in $D\left(\mathcal{A}^{\mathfrak{z}}\right)$.

Let $a_{\text {reg }}$ denote the restriction of $a: U \times(\mathfrak{z}+\mathfrak{u}) \rightarrow \mathfrak{z}+\mathfrak{u}$ to $U \times \mathfrak{z}$ reg. Then by Corollary $4.2, a_{\text {reg }}$ determines an isomorphism of varieties between $U \times \mathfrak{z}_{\text {reg }}$ and $\mathfrak{z}_{\text {reg }}+\mathfrak{u}$. Thus, if $1_{U}: U \rightarrow U$ is the identity morphism, then $a \circ\left(1_{U} \times\left.\eta\right|_{\mathfrak{z}_{\text {reg }}}\right) \circ$ ${\underset{\sim}{\text { reg }}}^{-1}: \mathfrak{z}_{\text {reg }}+\mathfrak{u} \rightarrow \mathfrak{z}+\mathfrak{u}$ is a morphism. Set $\widetilde{\phi}_{\text {reg }}=a \circ\left(1_{U} \times\left.\eta\right|_{\mathfrak{z} \text { reg }}\right) \circ a^{-1}$. Then $\widetilde{\phi}_{\mathrm{reg}}(\operatorname{Ad} u(t))=\operatorname{Ad} u(\eta(t))$ for $u$ in $U$ and $t$ in $\mathfrak{z}_{\text {reg }}$ so $\left.\widetilde{\phi}_{\text {reg }}\right|_{\mathfrak{z}_{\text {reg }}}=\left.\eta\right|_{\mathfrak{z} \text { reg }}$.

We next show that $\widetilde{\phi}_{\text {reg }}$ is $P$-equivariant. Then, since $\mathfrak{z}$ reg $+\mathfrak{u}$ is dense in $\mathfrak{z}+\mathfrak{u}$, it will follow that if $\widetilde{\phi}_{\text {reg }}$ extends to a morphism, $\widetilde{\phi}: \mathfrak{z}+\mathfrak{u} \rightarrow \mathfrak{z}+\mathfrak{u}$, then $\widetilde{\phi}$ is also $P$-equivariant. So suppose $p$ is in $P, t$ is in $\mathfrak{z}_{\text {reg }}$, and $n$ is in $\mathfrak{u}$. Then there is a $u$ in $U$ so that $t+n=\operatorname{Ad} u(t)$. Write $p u=u_{1} l$ where $u_{1}$ is in $U$ and $l$ is in $L$. Then

$$
\begin{aligned}
\widetilde{\phi}_{\text {reg }}(\operatorname{Ad} p(t+n)) & =\widetilde{\phi}_{\text {reg }}(\operatorname{Ad} p u(t)) \\
& =\widetilde{\phi}_{\text {reg }}\left(\operatorname{Ad} u_{1}(t)\right) \\
& =\operatorname{Ad} u_{1}(\eta(t)) \\
& =\operatorname{Ad} u_{1} l(\eta(t)) \\
& =\operatorname{Ad} p u(\eta(t)) \\
& =\operatorname{Ad} p\left(\widetilde{\phi}_{\text {reg }}(t+n)\right) .
\end{aligned}
$$

Therefore, $\widetilde{\phi}_{\text {reg }}$ is $P$-equivariant. 
Our strategy for showing that $\widetilde{\phi}_{\text {reg }}$ extends to all of $\mathfrak{z}+\mathfrak{u}$ is to show that $\widetilde{\phi}_{\text {reg }}$ extends to a morphism $\widetilde{\phi}_{i}: \mathfrak{z}^{i}+\mathfrak{u} \rightarrow \mathfrak{z}+\mathfrak{u}$ for all $\alpha$ in $\Phi_{\mathfrak{u}}$ where as in $\S 4, \mathfrak{z}^{i}=$ $\left\{t \in \mathfrak{z} \mid \beta(t) \neq 0\right.$ for all $\left.\beta \in \Phi_{\mathfrak{u}} \backslash \Psi_{i}\right\}$.

Assuming this has been done and that $\widetilde{\phi}_{\text {reg }}$ denotes the extension to $\bigcup_{i=1}^{s}\left(\mathfrak{z}^{i}+\mathfrak{u}\right)$, we can complete the proof as follows. If $f$ is in $k[\mathfrak{z}+\mathfrak{u}]$, then $f \widetilde{\phi}_{\text {reg }}$ is a rational function on $\mathfrak{z}+\mathfrak{u}$ whose domain contains $\bigcup_{i=1}^{s}\left(\mathfrak{z}^{i}+\mathfrak{u}\right)$. If $f \widetilde{\phi}_{\text {reg }}$ is not a regular function on $\mathfrak{z}+\mathfrak{u}$, then the set of points at which it's not defined is a closed subset of $\mathfrak{z}+\mathfrak{u}$ with codimension 1 . Now $\bigcup_{i=1}^{s}\left(\mathfrak{z}^{i}+\mathfrak{u}\right)$ is contained in the domain of $f \widetilde{\phi}_{\text {reg }}$ and so the set of points in $\mathfrak{z}+\mathfrak{u}$ at which $f \widetilde{\phi}_{\text {reg }}$ is not defined is contained in $(\mathfrak{z}+\mathfrak{u}) \backslash \bigcup_{i=1}^{s}\left(\mathfrak{z}^{i}+\mathfrak{u}\right)$. However

$$
(\mathfrak{z}+\mathfrak{u}) \backslash \bigcup_{i=1}^{s}\left(\mathfrak{z}^{i}+\mathfrak{u}\right)=\left(\bigcup_{i \neq j}\left(H_{i} \cap H_{j}\right)\right)+\mathfrak{u} .
$$

Thus, the set of points at which $f \widetilde{\phi}_{\text {reg }}$ is not defined has codimension at least 2 . Therefore $f \widetilde{\phi}_{\text {reg }}$ must be defined on all of $\mathfrak{z}+\mathfrak{u}$ and so $f \widetilde{\phi}_{\text {reg }}$ is in $k[\mathfrak{z}+\mathfrak{u}]$. It follows that $\widetilde{\phi}_{\text {reg }}^{\#}(k[\mathfrak{z}+\mathfrak{u}]) \subseteq k[\mathfrak{z}+\mathfrak{u}]$, and so $\widetilde{\phi}_{\text {reg }}$ extends to a morphism $\widetilde{\phi}: \mathfrak{z}+\mathfrak{u} \rightarrow \mathfrak{z}+\mathfrak{u}$.

It remains to show that $\widetilde{\phi}_{\text {reg }}$ can be extended to a morphism from $\mathfrak{z}^{i}+\mathfrak{u}$ to $\mathfrak{z}+\mathfrak{u}$ for $1 \leq i \leq s$. In order to show that $\widetilde{\phi}_{\text {reg }}$ extends to $\mathfrak{z}^{i}+\mathfrak{u}$, we'll finally use the hypothesis that $\eta$ is in $D\left(\mathcal{A}^{\mathfrak{z}}\right)$. Since $\eta$ is in $D\left(\mathcal{A}^{\mathfrak{z}}\right)$, for $\alpha$ in $\Phi_{\mathfrak{u}}, \eta^{\#}\left(\left.\alpha\right|_{\mathfrak{z}}\right)$ is a $k[\mathfrak{z}]$ multiple of $\left.\alpha\right|_{\mathfrak{z}}$. Say $\eta^{\#}\left(\left.\alpha_{i}\right|_{\mathfrak{z}}\right)=\left.f_{i} \alpha_{i}\right|_{\mathfrak{z}}$ for $1 \leq i \leq s$. Define $\eta_{i}: \mathfrak{z}^{i}+\mathfrak{u}_{i} \rightarrow \mathfrak{z}+\mathfrak{u}$ by $\eta_{i}(t+n)=\eta(t)+f_{i}(t) n$, so $\eta_{i}$ is a morphism. Recall that $a_{i}: U^{i} \times\left(\mathfrak{z}^{i}+\mathfrak{u}_{i}\right) \rightarrow \mathfrak{z}+\mathfrak{u}$ determines an isomorphism between $U^{i} \times\left(\mathfrak{z}^{i}+\mathfrak{u}_{i}\right)$ and $\mathfrak{z}^{i}+\mathfrak{u}$ by Proposition 4.3. Define $\widetilde{\phi}_{i}=a \circ\left(1_{U} \times \eta_{i}\right) \circ a_{i}^{-1}: \mathfrak{z}^{i}+\mathfrak{u} \rightarrow \mathfrak{z}+\mathfrak{u}$.

Lemma 5.2. The restriction of $\widetilde{\phi}_{i}$ to $\mathfrak{z}$ reg $+\mathfrak{u}$ is equal to $\widetilde{\phi}_{\text {reg }}$.

Proof. Suppose $t+n$ is in $\mathfrak{z r e g}+\mathfrak{u}$. Then $t+n=\operatorname{Ad} u(t)$ for some $u$ in $U$. Write $u=u^{i} u_{i}$ where $u^{i}$ is in $U^{i}$ and $u_{i}$ is in $U_{i}$. It follows from Proposition 4.4 that there is an $n_{1}$ in $\mathfrak{u}_{i}$ so that $\operatorname{Ad} u_{i}\left(t^{\prime}\right)=t^{\prime}+\alpha_{i}\left(t^{\prime}\right) n_{1}$ for every $t^{\prime}$ in $\mathfrak{z}$. Then $t+n=$ $\operatorname{Ad} u^{i}\left(t+\alpha_{i}(t) n_{1}\right)$ and so

$$
\begin{aligned}
\widetilde{\phi}_{i}(t+n) & =\operatorname{Ad} u^{i}\left(\eta(t)+f_{i}(t) \alpha_{i}(t) n_{1}\right) \\
& =\operatorname{Ad} u^{i}\left(\eta(t)+\alpha_{i}(\eta(t)) n_{1}\right) \\
& =\operatorname{Ad} u^{i} u_{i}(\eta(t)) \\
& =\operatorname{Ad} u(\eta(t)) \\
& =\widetilde{\phi}_{\text {reg }}(t+n) .
\end{aligned}
$$

This completes the proof of the lemma.

It follows from the lemma that $\widetilde{\phi}_{\text {reg }}$ extends to $\mathfrak{z}^{i}+\mathfrak{u}$ for $\alpha$ in $\Phi_{\mathfrak{u}}$, and so the proof of Theorem 5.1 is complete.

Corollary 5.3. The graded $k[\mathfrak{z}]$-modules, $\operatorname{Hom}_{G}\left(\mathfrak{g}^{*}, k\left[G \times{ }^{P}(\mathfrak{z}+\mathfrak{u})\right]\right)$ and $D\left(\mathcal{A}^{\mathfrak{z}}\right)$ are isomorphic.

Proof. It is easily seen that the natural map between $\operatorname{Hom}_{G}\left(\mathfrak{g}^{*}, k\left[G \times{ }^{P}(\mathfrak{z}+\mathfrak{u})\right]\right)$ and $\operatorname{Mor}_{G}\left(G \times{ }^{P}(\mathfrak{z}+\mathfrak{u}), \mathfrak{g}\right)$ is an isomorphism of graded $k[\mathfrak{z}]$-modules, and so if $\Gamma$ is the 
composition of $\Lambda$ with this isomorphism, then

$$
\Gamma: \operatorname{Hom}_{G}\left(\mathfrak{g}^{*}, k\left[G \times{ }^{P}(\mathfrak{z}+\mathfrak{u})\right]\right) \rightarrow D\left(\mathcal{A}^{\mathfrak{z}}\right)
$$

is an isomorphism of graded $k[\mathfrak{z}]$-modules.

\section{Conclusions}

As in $\S 5$, since $P$ acts trivially on $(\mathfrak{z}+\mathfrak{u}) / \mathfrak{u}$, we can take $W=\mathfrak{z}+\mathfrak{u}, W_{0}=\mathfrak{z}$, and $W_{1}=\mathfrak{u}$ in Lemma 3.3 and conclude that there is an isomorphism of graded $k[\mathfrak{z}]$-modules, $k[\mathfrak{z}] \otimes \operatorname{Hom}_{G}\left(\mathfrak{g}^{*}, k\left[G \times{ }^{P} \mathfrak{u}\right]\right) \cong \operatorname{Mor}_{G}\left(G \times{ }^{P}(\mathfrak{z}+\mathfrak{u}), \mathfrak{g}\right)$. Composing this isomorphism with the isomorphism of Theorem 5.1 and using that $\mathfrak{g}$ affords a self-dual representation of $G$, we obtain an isomorphism of graded $k[\mathfrak{z}]$-modules, $k[\mathfrak{z}] \otimes \operatorname{Hom}_{G}\left(\mathfrak{g}, k\left[G \times{ }^{P} \mathfrak{u}\right]\right) \cong D\left(\mathcal{A}^{\mathfrak{z}}\right)$. It follows that $\left(\mathfrak{z}, \mathcal{A}^{\mathfrak{z}}\right)$ is a free hyperplane arrangement. We can now prove the following result of Orlik and Terao [8].

Corollary 6.1. If $(V, \mathcal{A})$ is a reflection arrangement arising from a finite Weyl group, then for $X$ in the lattice of $\mathcal{A}$, the restricted arrangement, $\left(X, \mathcal{A}^{X}\right)$ is free.

Proof. Suppose $\Phi$ is a root system in a $k$-vector space $V$. Let $\mathcal{A}$ be the arrangement of hyperplanes in $V$ consisting of the hyperplanes orthogonal to the vectors in $\Phi$. It's straightforward to check that if $G$ is the adjoint group with root system $\Phi=\Phi(G, T)$, then every restriction of $(V, \mathcal{A})$ arises as $\left(\mathfrak{z}, \mathcal{A}^{\mathfrak{z}}\right)$ for a suitable choice of $P$ and $L$.

Alternately, if $W$ is the Weyl group of $(V, \mathcal{A})$, then $W$ acts on the lattice of $\mathcal{A}$ and it follows from standard results about root systems that if $\Pi$ is a base of $\Phi$, then every $W$-orbit in the lattice of $\mathcal{A}$ contains a representative that's an intersection of the hyperplanes orthogonal to the vectors in a subset of $\Pi$ (see [6]). Clearly, if $X$ is in the lattice of $\mathcal{A}$ and $w$ is in $W$, then $\mathcal{A}^{X}$ is free if and only if $\mathcal{A}^{w X}$ is free. Now if $G$ is the adjoint group with root system $\Phi=\Phi(G, T), B$ is a Borel subgroup of $G$ containing $T, P$ is a parabolic subgroup of $G$ containing $B$, and $L$ is the standard Levi factor in $P$, then $\left(\mathfrak{z}, \mathcal{A}^{\mathfrak{z}}\right)$ is free. Since every orbit of $W$ on the lattice of $\mathcal{A}$ contains a representative of this form, the result follows.

Finally, we show that the isomorphism of graded $k[\mathfrak{z}]$-modules between $k[\mathfrak{z}] \otimes$ $\operatorname{Hom}_{G}\left(\mathfrak{g}, k\left[G \times{ }^{P} \mathfrak{u}\right]\right)$ and $D\left(\mathcal{A}^{\mathfrak{z}}\right)$ can be used to prove the result of Sommers, Trapa, and Broer mentioned in the introduction.

Corollary 6.2. The multiplicity of the adjoint representation of $G$ in the graded representation $k\left[G \times{ }^{P} \mathfrak{u}\right]$ is given by

$$
\sum_{j \geq 0} \operatorname{dim} \operatorname{Hom}_{G}\left(\mathfrak{g}, k\left[G \times{ }^{P} \mathfrak{u}\right]_{j}\right) q^{j}=q^{n_{1}^{3}}+\cdots+q^{n_{r}^{3}}
$$

where $q$ is an indeterminate and $\left\{n_{1}^{\mathfrak{z}}, \ldots, n_{r}^{\mathfrak{z}}\right\}$ is the multiset of exponents of $\mathcal{A}^{\mathfrak{z}}$.

Proof. As in $\S 2, \operatorname{Hom}_{G}\left(\mathfrak{g}, k\left[G \times{ }^{P} \mathfrak{u}\right]\right)$ inherits a grading from the scalar action of $k$ on $\mathfrak{u}$. Suppose $\left\{\phi_{1}, \ldots, \phi_{s}\right\}$ is a homogeneous basis of $\operatorname{Hom}_{G}\left(\mathfrak{g}, k\left[G \times{ }^{P} \mathfrak{u}\right]\right)$ with $\phi_{i}$ in $\operatorname{Hom}_{G}\left(\mathfrak{g}, k\left[G \times{ }^{P} \mathfrak{u}\right]_{d_{i}}\right)$ for $1 \leq i \leq s$. Then clearly

$$
\sum_{j \geq 0} \operatorname{dim} \operatorname{Hom}_{G}\left(\mathfrak{g}, k\left[G \times{ }^{P} \mathfrak{u}\right]_{j}\right) q^{j}=q^{d_{1}}+\cdots+q^{d_{s}} .
$$

On the other hand, the set $\left\{1 \otimes \phi_{1}, \ldots, 1 \otimes \phi_{s}\right\}$ is a homogeneous basis of $k[\mathfrak{z}] \otimes$ $\operatorname{Hom}_{G}\left(\mathfrak{g}, k\left[G \times{ }^{P} \mathfrak{u}\right]\right)$ and so corresponds to a homogeneous basis of $D\left(\mathcal{A}^{\mathfrak{z}}\right)$ under 
the isomorphism $k[\mathfrak{z}] \otimes \operatorname{Hom}_{G}\left(\mathfrak{g}, k\left[G \times{ }^{P} \mathfrak{u}\right]\right) \cong D\left(\mathcal{A}^{\mathfrak{z}}\right)$. Therefore, the multisets $\left\{d_{1}, \ldots, d_{s}\right\}$ and $\left\{n_{1}^{\mathfrak{z}}, \ldots, n_{r}^{\mathfrak{z}}\right\}$ are equal.

\section{REFERENCES}

[1] W. Borho. Über Schichten halbeinfacher Lie-Algebren. Invent. Math., 65:283-317, 1981. MR 83b:17006

[2] A. Broer. The sum of generalized exponents and Chevalley's restriction theorem for modules of covariants. Indag. Math., 6:385-396, 1995. MR 96j:20058

[3] A. Broer. Lectures on decomposition classes. In Representation Theory and Algebraic Geometry, Proceedings of the NATO Advanced Study Institute, Montreal, Canada, July 28 - August 8, 1997 (A. Broer, editor). Kluwer, 1998. MR 99:03

[4] A. Broer. Hyperplane arrangements, Springer representations, and exponents. In Advances in Geometry, volume 172 of Progress in Mathematics (J. Brylinski, editor). Birkhäuser, 1999. MR 99:07

[5] B. Kostant. Lie group representations on polynomial rings. Amer. J. Math., 85:327-404, 1963. MR 28:1252

[6] P. Orlik and L. Solomon. Coxeter arrangements. In Singularities, volume 40 of Proc. Symp. Pure Math., pages 269-292, Amer. Math. Soc., 1983. MR 85b:32016

[7] P. Orlik and H. Terao. Arrangements of Hyperplanes. Springer-Verlag, 1992. MR 94e:52014

[8] P. Orlik and H. Terao. Coxeter arrangements are hereditarily free. Tôhoku Math. J., 45:369383, 1993. MR 94h:52023

[9] E. Sommers and P. Trapa. The adjoint representation in rings of functions. Represent. Theory, 1:182-189, 1997. MR 98i:22020

Department of Mathematics, University of North Texas, Denton, Texas 76203

E-mail address: douglass@unt.edu 